\title{
Fabrication of Upconverting Hybrid Nanoparticles for Near-Infrared Light Triggered Drug Release
}

\author{
Ranran Zhang, ${ }^{1}$ Risheng Yao, ${ }^{1}$ Binbin Ding, ${ }^{1}$ Yuxin Shen, ${ }^{1}$ Shengwen Shui, \\ Lei Wang, ${ }^{1}$ Yu Li, ${ }^{2}$ Xianzhu Yang, ${ }^{1}$ and Wei Tao ${ }^{1}$ \\ ${ }^{1}$ School of Medical Engineering, Hefei University of Engineering, Hefei, Anhui, China \\ ${ }^{2}$ School of Material Science and Engineering, Wuhan University of Technology, Wuhan, Hubei, China \\ Correspondence should be addressed to Risheng Yao; yaors@hfut.edu.cn and Wei Tao; taow@hfut.edu.cn
}

Received 10 April 2014; Accepted 26 May 2014; Published 21 July 2014

Academic Editor: Haisheng Qian

Copyright (C) 2014 Ranran Zhang et al. This is an open access article distributed under the Creative Commons Attribution License, which permits unrestricted use, distribution, and reproduction in any medium, provided the original work is properly cited.

Low tissue penetration and harmful effects of (ultraviolet) UV or visible light on normal tissue limit exploiting nanocarriers for the application of light-controlled drug release. Two strategies may solve the problem: one is to improve the sensitivity of the nanocarriers to light to decrease the radiation time; the other one is using more friendly light as the trigger. In this work, we fabricated a core-shell hybrid nanoparticle with an upconverting nanoparticle (UCNP) as the core and thermo- and light-responsive block copolymers as the shell to combine the two strategies together. The results indicated that the sensitivity of the block copolymer to light could be enhanced by decreasing the photolabile moieties in the polymer, and the UCNP could transfer near-infrared (NIR) light, which is more friendly to tissue and cell, to UV light to trigger the phase conversion of the block polymers in situ. Using Nile Red (NR) as the model drug, the hybrid nanoparticles were further proved to be able to act as carriers with the character of NIR triggered drug release.

\section{Introduction}

Stimuli responsive nanoparticles are attracting more and more attentions because of their potential for controlled drug delivery applications [1-5]. Among the various stimuli, light is a typical external stimulus and has many superior characters. For example, light can be easily controlled and regulated by external apparatuses, which will offer the temporal and spatial selectivity [6-10]; light can induce various photochemical reactions, which makes it easy to exploit diversiform photosensitive materials [11-14]. However, the tissue penetration of light is low, and providing enough photons to the stimuli responsive nanoparticles in deep tissue is difficult. Furthermore, most of photochemical reactions are induced by UV or visible light, whose harmful effects on normal tissue and health cells limit their applications in lightcontrolled drug release.

Comparing to UV and visible light, NIR light is more ideal because of its deeper penetration into tissue and less detrimental to healthy cells [15-17]. However, as mentioned above, most of photochemical reactions require high-energy UV or visible light. Recently, UCNPs have emerged as a powerful tool to convert NIR light to higher-energy light in the UV, visible, or NIR regions [18-20]. When UCNPs are incorporated in UV sensitive materials, NIR light may be able to induce the photoreaction of the UV sensitive materials in deeper tissues and decrease the damage in the radiation path from radiation source to the target tissue [21-25].

Another way to overcome the drawbacks of light is to increase the sensitivity of material to light, because less requirement for photons means less radiation time of light or more efficient photochemical reaction in deeper regions. Internal stimuli are biologic properties of disease areas, such as temperature $[26,27], \mathrm{pH}[28,29]$, and redox potential $[30,31]$, which generally are stable in the body. Therefore, when internal stimuli sensitive materials combine with a small amount of photosensitive moieties, the effect of photoreaction may be amplified by the internal stimuli [14]. For example, poly(N-isopropylacrylamide) (PNIPAM) is a typical thermosensitive polymer, which is soluble in water 


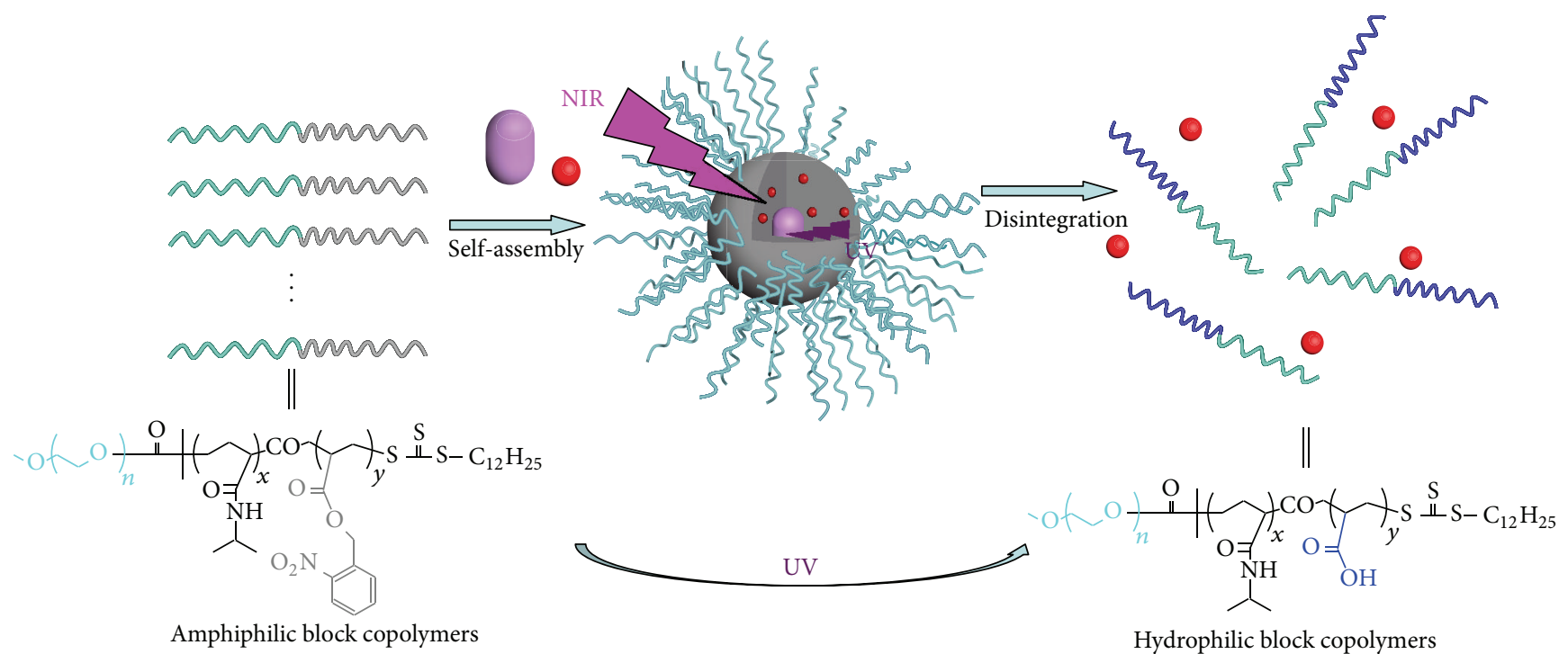

Nile Red

UCNP

Scheme 1: Schematic illustration for fabrication of the core-shell hybrid nanoparticle and NIR triggered release of the loaded model drugs (Nile Red).

below its lower critical temperature (LCST) at about $32^{\circ} \mathrm{C}$ but insoluble above it. When NIPAMs are copolymerized with hydrophobic or hydrophilic monomers, the LCST of the copolymers will accordingly decrease or increase [32]. Based on the property of PNIPAM, Ionov and Diez synthesized photoresist material by copolymerizing NIPAMs and nitrobenzyl acrylates (NBAs) [33]. They found that only $5 \mathrm{~mol} \%$ of NBA would decrease the LCST of the copolymer below room temperature $\left(\sim 10^{\circ} \mathrm{C}\right)$ because of the hydrophobic nature of the NBA. Upon irradiation of UV light at $365 \mathrm{~nm}$, the NBA moieties left and the residual hydrophilic carboxyl groups increased the LCST of the copolymer above $40^{\circ} \mathrm{C}$. This character of PNIPAM also was used for photocontrolled drug release. Recently, Li et al. prepared thermo- and lightresponsive micelles as drug carriers by self-assembling PEO$b$-P(NIPAM-co-NBA) in water [34]. Upon the irradiation of UV light, the micelles were disintegrated because of the LCST change of the P(NIPAM-co-NBA) block and realized UVtriggered drug release.

To combine the advantages of the less detriment of NIR light to body and enhanced sensitivity of thermo- and lightresponsive polymers to light, we fabricated upconverting hybrid nanoparticles by self-assembling PEO- $b$-P(NIPAMco-NBA) with the presence of UCNPs in water. The results indicated that the hybrid nanoparticles had a core-shell structure with UCNP as the core and PEO- $b$-P(NIPAM-co-NBA) as the shell. Furthermore, we used Nile Reds as the model drugs to investigate their light induced release. The result indicated that, as shown in Scheme 1, when the upconverting hybrid nanoparticles were irradiated by NIR light, the UCNPs converted NIR to UV light to induce the photocleavage reaction of NBA moieties in situ; then, as a result of the LCST change of the block copolymers, nanoparticles disintegrated and released the loaded NR molecules.

\section{Materials and Methods}

2.1. Materials. Poly(ethylene oxide) monomethyl ether (PEO, Mn: 1000, $\left.M_{w} / M_{n}: 1.05\right)$ was purchased from Alfa Aesar and used as received. N-Isopropylacrylamide (NIPAM, TCI) was recrystallized twice from hexanes. Azobisisobutyronitrile (AIBN, Sinopharm Chemical Reagent, 95\%) was recrystallized from 95\% ethanol. Triethylamine (TEA, Aldrich) and dichloromethane (DCM, Sinopharm Chemical Reagent) were dried over $\mathrm{CaH}_{2}$. Acryloyl chloride, 2-nitrobenzyl alcohol, N, $\mathrm{N}^{\prime}$-dicyclohexylcarbodiimide (DCC, 99\%), and N,N ${ }^{\prime}$-dimethylaminopyridine (DMAP, 98\%) were purchased from Aldrich. Acetone, carbon disulfide, 1-dodecanethiol, isopropanol, tetrabutyl ammonium bromide, chloroform, hexane, and diethyl ether were purchased from Sinopharm Chemical Reagent (AR) and used without further purification. 2-Nitrobenzyl acrylate (NBA) [33], S-1-dodecyl-S $\left(\alpha, \alpha^{\prime}\right.$ dimethyl- $\alpha^{\prime \prime}$-acetic acid) trithiocarbonate (TTCA) [35], PEO-TTCA, and $\beta-\mathrm{NaYF}_{4}: \mathrm{Tm}^{3+} 0.5 \mathrm{~mol} \%, \mathrm{Yb}^{3+} 30 \mathrm{~mol} \%$ upconverting nanoparticles (UCNPs) [18] were synthesized according to previously reported literature procedures. Water was deionized with a Millipore system to a resistivity of $18.2 \mathrm{M} \Omega \cdot \mathrm{cm}$. All reagents were used as received unless otherwise stated. 
2.2. Synthesis of the Amphiphilic $P E O_{1000}-b-P(N I P A M-c o-$ NBA) Block Copolymers. In this work, two $\mathrm{PEO}_{1000}-b$ $\mathrm{P}$ (NIPAM-co-NBA) block copolymers with different NBA contents were synthesized in 1,4-dioxane with the $\mathrm{PEO}_{1000^{-}}$ TTCA as the macroRAFT agent and AIBN as the initiator. The $\left[\mathrm{PEO}_{1000}-\mathrm{TTCA}\right]:[\mathrm{NIPAM}+\mathrm{NBA}]:[\mathrm{AIBN}]$ ratio was maintained at $1: 180: 0.2$ (mole basis) while the [NIPAM] : [NBA] ratio was $11: 1$ and $20: 1$, respectively. The polymerization procedure was as follows. NIPAM, NBA, AIBN, $\mathrm{PEO}_{1000}$-TTCA, and 1,4-dioxane were charged into a round-bottom flask equipped with a magnetic stirring bar. After being sealed with a rubber septum and then deoxygenated with a stream of bubbled nitrogen for $30 \mathrm{~min}$, the flask was immersed in an oil bath at $70^{\circ} \mathrm{C}$ and stirred for $24 \mathrm{~h}$. Then, the mixture was cooled to room temperature and precipitated into an excess of diethyl ether. The precipitate was collected and dried under vacuum for $24 \mathrm{~h}$ at room temperature to afford the product.

\subsection{Preparation of Polymer Micelles and Encapsulation of} Nile Red. The procedure for preparation of polymer micelles was as follows. $10 \mathrm{mg}$ of the block copolymer (BCP) was dissolved in $1 \mathrm{~mL}$ of THF, which is a good solvent for the two blocks. Under vigorous stirring, deionized water was then added slowly until the solution exhibited a bluish tinge, which indicated the formation of the micelles. After $2 \mathrm{~h}$ of stirring, a 4 -fold volume of water was added for quenching of micelles. THF and most of water were then removed by evaporation under vacuum at $30^{\circ} \mathrm{C}$ until the solution regained the initial concentration. BCP micelles with encapsulated NR were prepared by the procedure analogue to the one for $\mathrm{BCP}$ micelles. In brief, the BCP and NR were first dissolved in THF at a concentration of $0.8 \mathrm{mg} / \mathrm{mL}$ and $0.125 \mathrm{mg} / \mathrm{mL}$. Water was then added to induce the formation of micelles and simultaneous encapsulation of NR by the hydrophobic core of the micelles. After $2 \mathrm{~h}$ of stirring, a 4 -fold volume of water was added for quenching of micelles and precipitation of unloaded NR. Precipitated NR was removed by filtration through $0.45 \mu \mathrm{m}$ membrane, and THF and most of water were evaporated by evaporation under vacuum at $30^{\circ} \mathrm{C}$ until the solution regained the initial concentration.

2.4. Preparation of the NIR Responsive Hybrid Nanoparticles. The NIR responsive hybrid nanoparticles were prepared as follows. BCP $(8 \mathrm{mg})$ and UCNPs $(8 \mathrm{mg})$ were dissolved in THF $(5 \mathrm{~mL})$. Under vigorous stirring at room temperature, $40 \mathrm{~mL}$ of deionized water was then added dropwise to the solution over $2 \mathrm{~h}$. The obtained mixture was centrifuged at $4800 \mathrm{rpm}$ for $10 \mathrm{~min}$. The supernatant was discarded and the precipitate was redispersed in deionized water $(10 \mathrm{~mL})$ under mild stirring to afford the solution of the hybrid nanoparticles. Nile Red loaded hybrid nanoparticles were prepared by the analogue method mentioned above. In brief, the THF solution of Nile Red $(10 \mathrm{mg} / \mathrm{mL}, 25 \mu \mathrm{L})$ was added into $5 \mathrm{~mL}$ of THF solution containing $8 \mathrm{mg}$ of BCP and $8 \mathrm{mg}$ of UCNPs. Then, $40 \mathrm{~mL}$ of deionized water was added dropwise over $2 \mathrm{~h}$. After the solution was centrifuged at $4800 \mathrm{rpm}$ for $10 \mathrm{~min}$, the supernatant was discarded. Finally, the precipitate was redispersed in deionized water $(10 \mathrm{~mL})$ under mild stirring to afford the solution of the NR loaded hybrid nanoparticles.

\subsection{Determination of Critical Micellization Concentration} $(C M C)$. The fluorescence probe method was employed to determine the CMC of the micelles as follows [36]. A predetermined amount of pyrene stock solution in acetone was added into an empty vial, and then the acetone was evaporated completely. A calculated amount of copolymer solutions at varying concentrations was added to the vial, and the final concentration of pyrene in each flask was fixed at 6.0 $\times 10^{-7} \mathrm{~mol} \mathrm{~L}^{-1}$. The excitation spectra were recorded at $25^{\circ} \mathrm{C}$ on a spectrofluorophotometer with $\lambda_{\mathrm{em}}$ at $390 \mathrm{~nm}$ and a slit width of $5 \mathrm{~nm}$.

\section{Characterization}

${ }^{1} \mathrm{H}$ NMR spectra were characterized in $\mathrm{CDCl}_{3}$ by VNMRS600 spectrometer $(600 \mathrm{MHz})$. The molecular weight and molecular weight distribution of the polymer were determined by gel permeation chromatography (GPC), THF was used as eluent (elution rate, $1.0 \mathrm{~mL} \mathrm{~min}^{-1}$ ), and polystyrene standards were employed for calibration. Dynamic light scattering (DLS) measurements were carried out using a Zetasizer (Nano-ZS 90, Malvern Instruments), and these samples were averaged over three measurements. Transmission electron microscopy (TEM) observations were conducted on a JEM-2100F electron microscope. The sample was prepared on copper grids coated with thin films of formvar and carbon successively. Fourier transform infrared (FT-IR) spectra were recorded on a Nicolet 67 spectrometer. UV-vis spectra were acquired on Hitachi U-5100 spectrophotometer. Fluorescence emission spectra were recorded using Hitachi F-2700 spectrofluorometer. The slit widths were both kept at $5 \mathrm{~nm}$ for excitation and emission, and scanning speed was set at $300 \mathrm{~nm} / \mathrm{min}$. UV-vis spot curing system (Futansi) was used to study the photoinduced dissociation of polymer micelles and the release of encapsulated NR. The micelle solutions were exposed to UV light under stirring. All samples were filtered through $0.45 \mu \mathrm{m}$ filter before the measurement to remove dust.

\section{Result and Discussion}

4.1. Synthesis of PEO-b-P(NIPAM-co-NBA) Diblock Copolymers. Two PEO- $b$-P(NIPAM-co-NBA) block copolymers (denoted as $\mathrm{p} 1$ and $\mathrm{p} 2$ ) with different NBA moieties were synthesized by RAFT polymerizations using $\mathrm{PEO}_{1000}$-TTCA as the macroRAFT agent. GPC analysis showed that the molecular weights of p1 and p2 were 23.7 and $23.4 \mathrm{kDa}$, respectively, and the corresponding polydispersities indexes (PDI) were 1.62 and 1.59. The monomodal and symmetric elution peaks of the products clearly shifted to the higher molecular weight region in comparison with that of PEO macroRAFT agent (Figure 1(a)), indicating that block copolymers were successfully synthesized. The chemical structures 


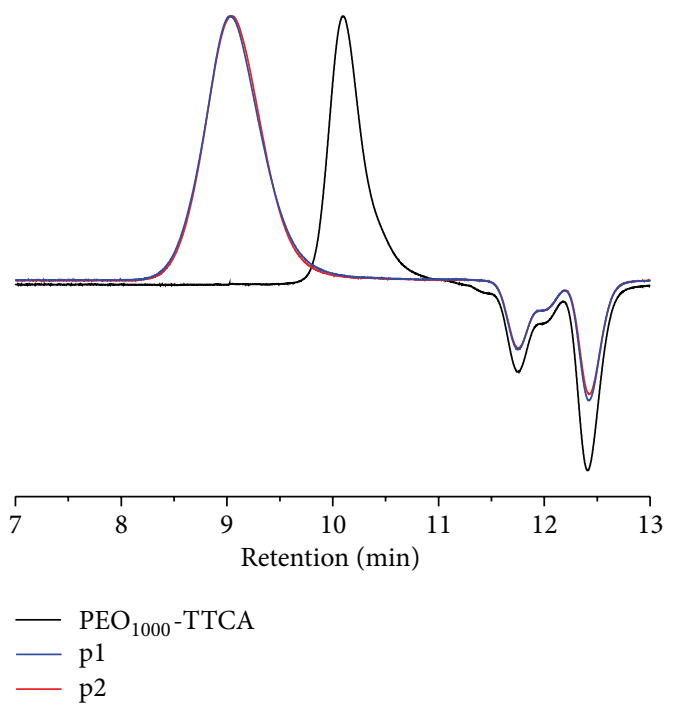

(a)
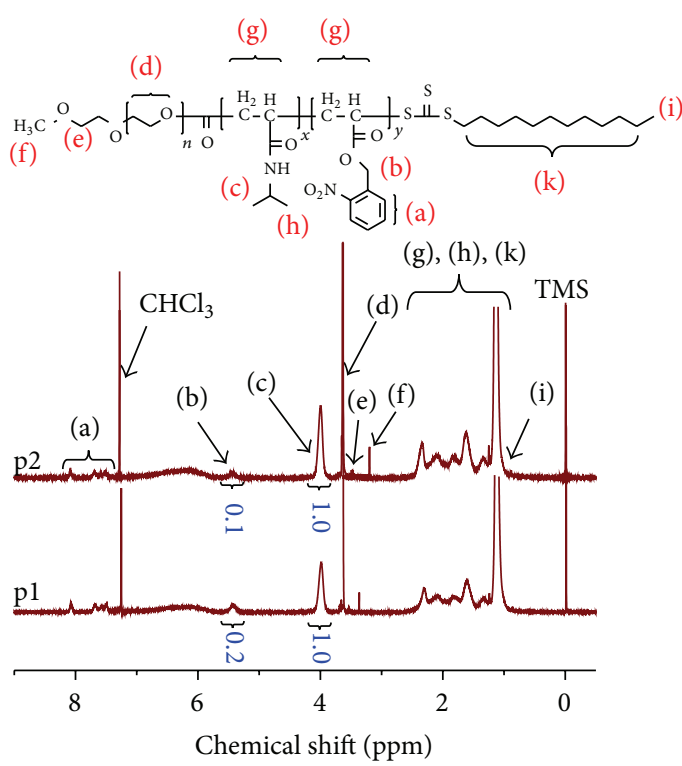

(b)

FIGURE 1: (a) GPC curves of PEO macroinitiator and the two block copolymers with different $\mathrm{NBA}$ moieties; (b) $\left.{ }^{1} \mathrm{H} \mathrm{NMR} \mathrm{(in} \mathrm{CDCl} \mathrm{N}_{3}\right) \operatorname{spectra}$ of $\mathrm{p} 1$ and $\mathrm{p} 2$.

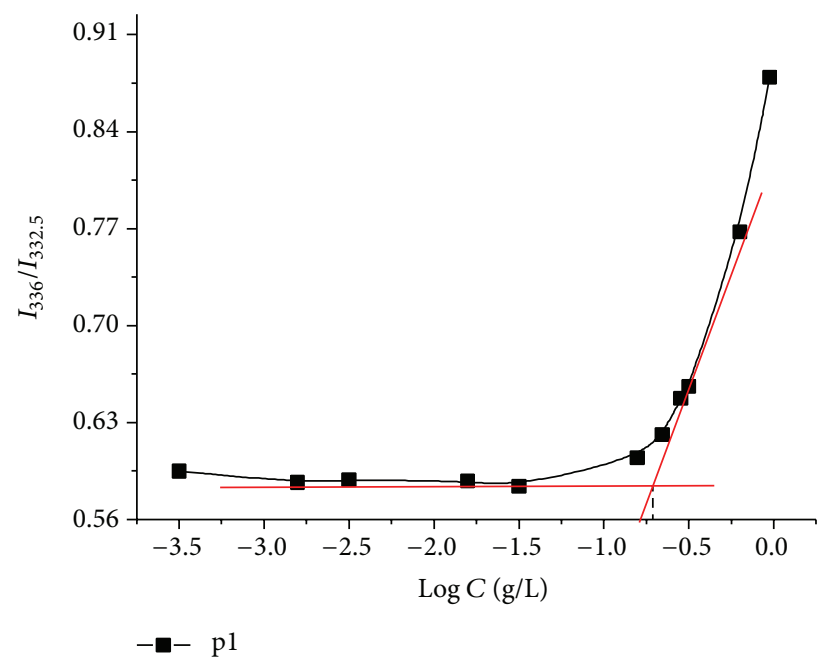

(a)

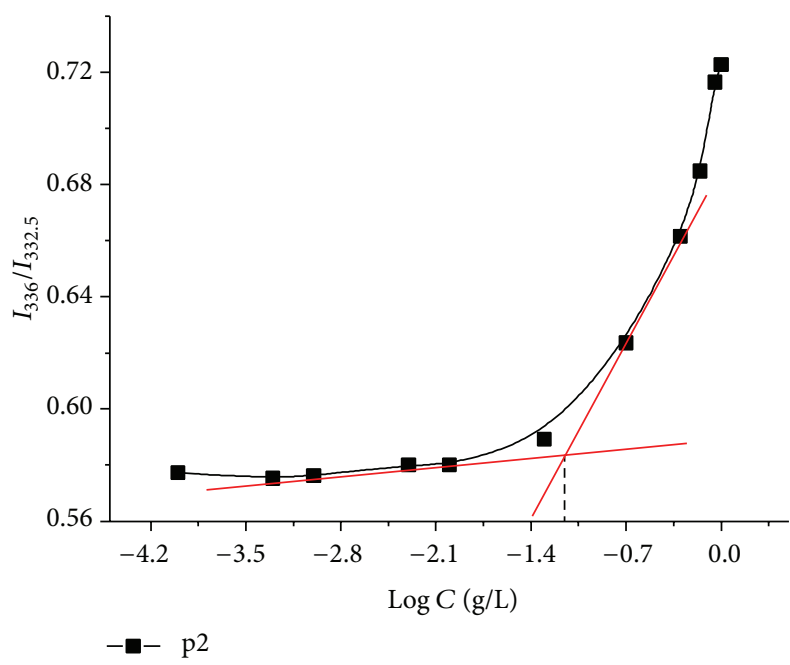

(b)

FIGURE 2: Plots of intensity ratio $\left(I_{336} / I_{332.5}\right)$ from the pyrene excitation spectra as a function of concentrations of p1 (a) and p2 (b), respectively. Pyrene concentration was fixed to be $6.0 \times 10^{-7} \mathrm{M}$, and the temperature is at $25^{\circ} \mathrm{C}$.

of the polymers were also characterized by ${ }^{1} \mathrm{H}$ NMR. As shown in Figure 1(b), the peaks at $3.37 \mathrm{ppm}, 3.98 \mathrm{ppm}$, and $5.42 \mathrm{ppm}$ are characteristic proton signals of $\mathrm{PEO}\left(-\mathrm{CH}_{2}-\right.$ $\mathrm{CH}_{2}-\mathrm{O}-$ ), NBA (benzyl $\mathrm{CH}_{2}$ protons), and NIPAM ($\left.\mathrm{CH}\left(\mathrm{CH}_{3}\right)_{2}\right)$ moieties, respectively. The NBA molar contents in $\mathrm{P}$ (NIPAM-co-NBA) block of $\mathrm{p} 1$ and $\mathrm{p} 2$ were calculated according to the equation of $0.5 \times \mathrm{A}_{5.42}: \mathrm{A}_{3.98}$, where the $\mathrm{A}_{5.42}$ and $\mathrm{A}_{3.98}$ are areas of the peaks at 5.42 and $3.98 \mathrm{ppm}$, respectively, and the results were 5.06 and $9.41 \mathrm{~mol} \%$. The molecular weights of p1 and p2 were also calculated according to ${ }^{1} \mathrm{H}$ $\mathrm{NMR}$; the results were $23.5 \mathrm{kDa}$ and $23.4 \mathrm{kDa}$, respectively, which coincided with the results obtained by GPC.

4.2. Micellization of PEO-b-P(NIPAM-co-NBA) and UVTriggered Micellar Disintegration in Aqueous Media. NBA moieties in P(NIPAM-co-NBA) block are in the form of $o$ nitrobenzyl (o-NB) ester. Only $5 \mathrm{~mol} \%$ of $o$-NB moieties will decrease the LCST of the block copolymer below $10^{\circ} \mathrm{C}$, and 


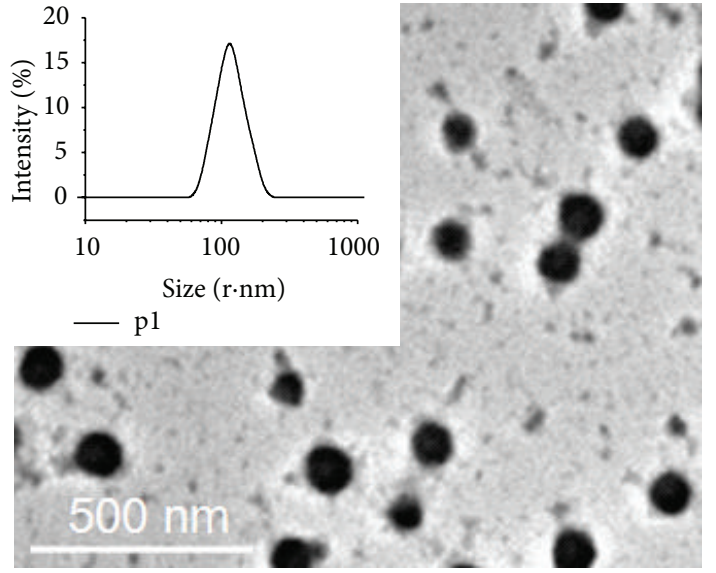

(a)
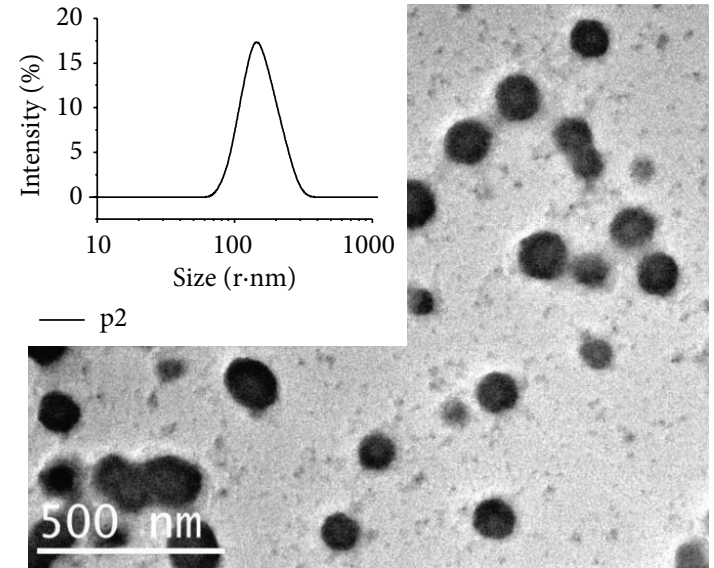

(b)

FIGURE 3: High-resolution TEM micrographs of the self-assemblies of (a) p1 and (b) p2. Insets are corresponding hydrodynamic diameter distributions recorded by DLS. Note. Staining was performed with phosphotungstic acid and the tiny particles may be the crystal of excess phosphotungstic acid.

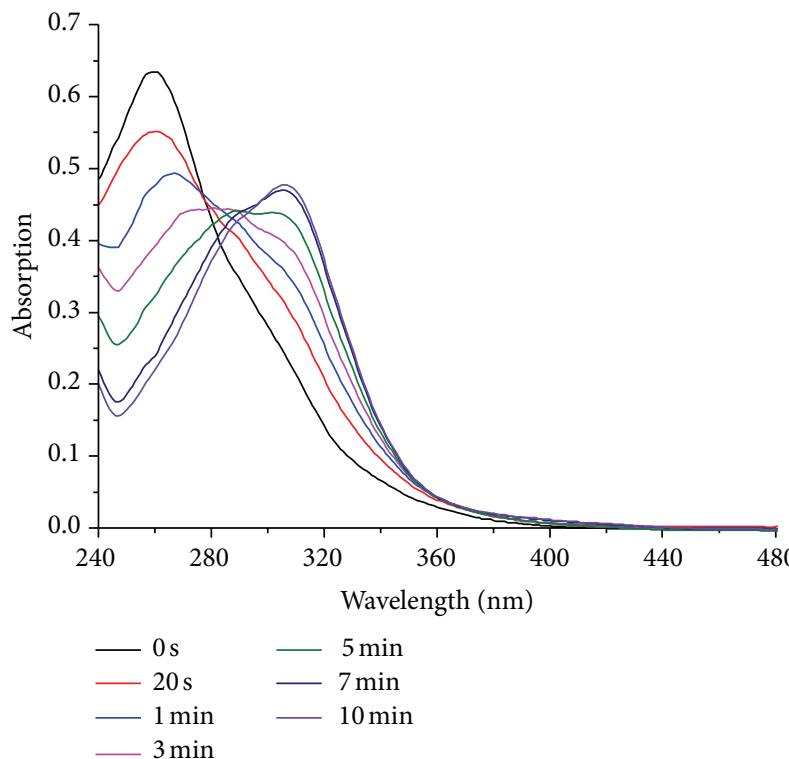

(a)

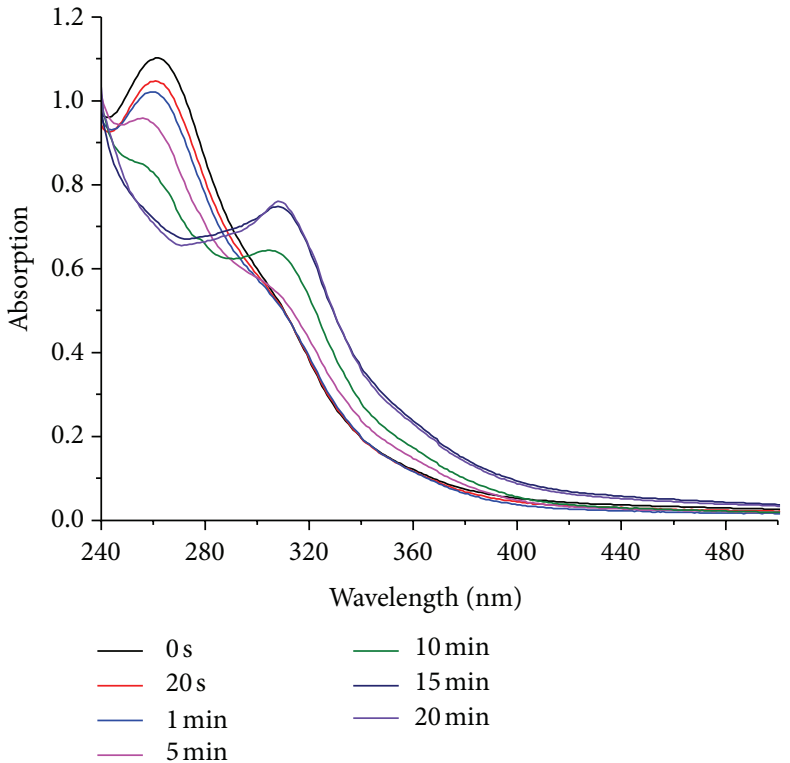

(b)

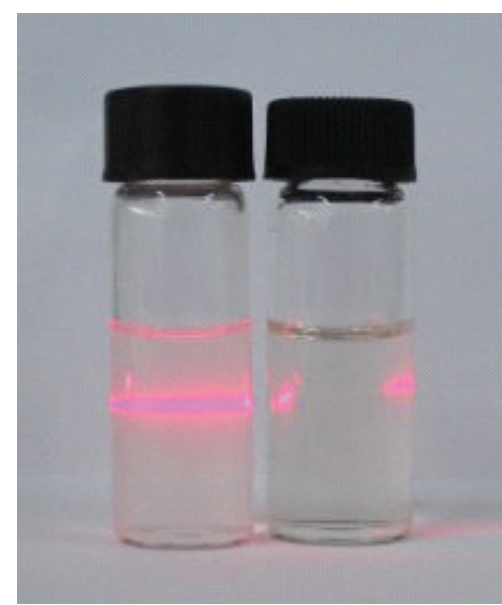

(c)

FIGURE 4: UV-vis spectra of (a) p1 micellar solution and (b) p2 micellar solution in water after various UV irradiation time; (c) photograph of the Tyndall effect of the p1 solution $(0.8 \mathrm{mg} / \mathrm{mL})$ before (left) and after (right) irradiation of UV light at $365 \mathrm{~nm}\left(2000 \mathrm{~mW} / \mathrm{cm}^{2}\right)$ for $10 \mathrm{~min}$. 


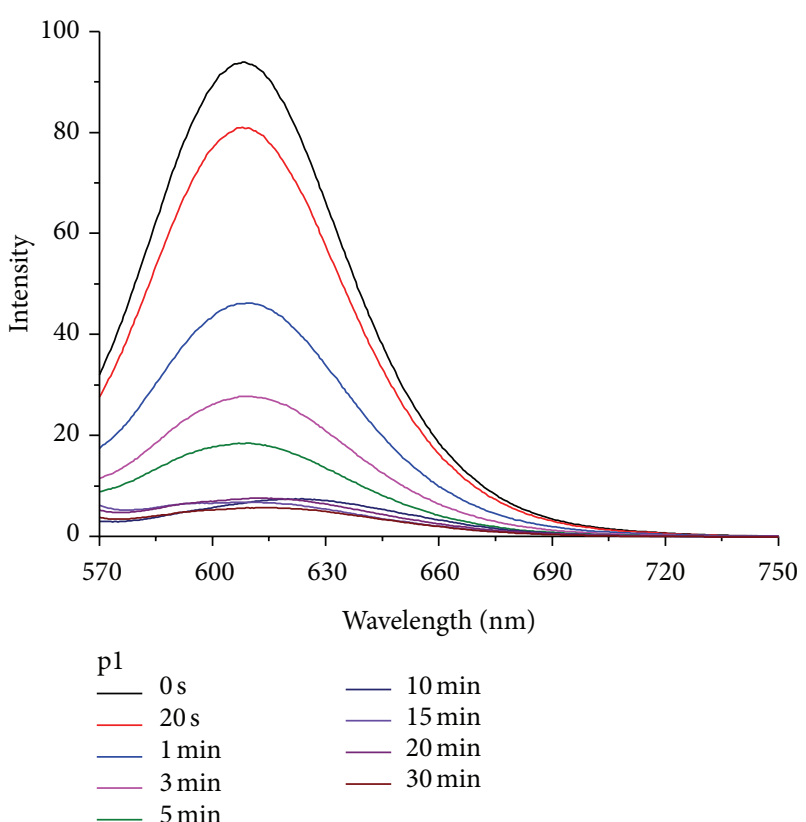

(a)

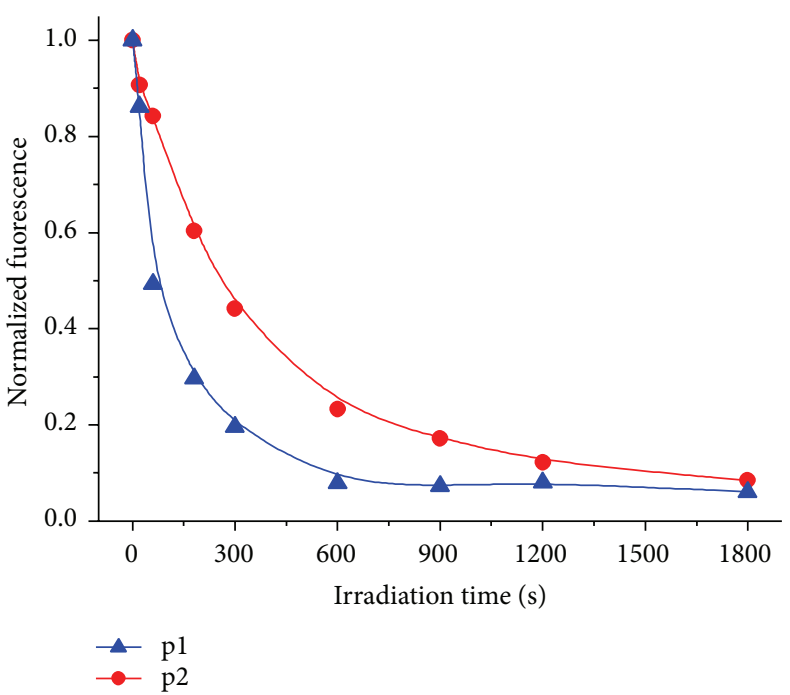

(b)

Figure 5: (a) Evolution of fluorescence emission spectra $\left(\lambda_{\text {ex }}=550 \mathrm{~nm}\right)$ of NR loaded PEO- $b$-P(NIPAM-co-NBA) diblock copolymer micelles under various UV irradiation time $\left(2000 \mathrm{~mW} / \mathrm{cm}^{2} ; 0.8 \mathrm{mg} / \mathrm{mL}, 2 \mathrm{~mL}\right)$; (b) plots of normalized fluorescence intensity versus UV light irradiation time for the NR loaded micelles of the two block copolymers in aqueous solution.

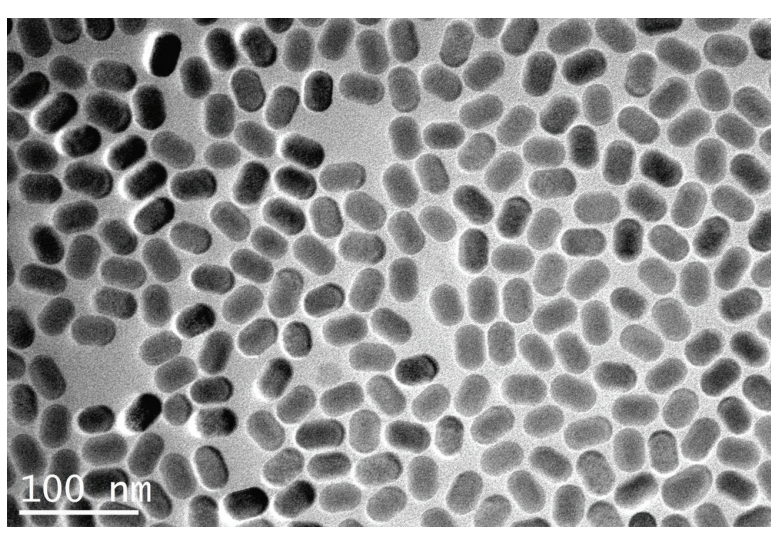

(a)

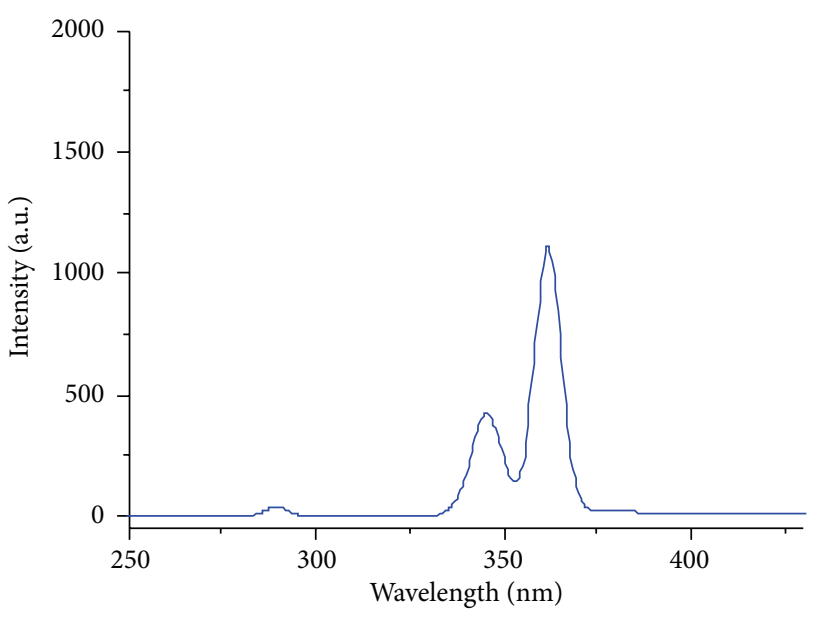

(b)

FIGURE 6: (a) TEM micrographs of the $\mathrm{NaYF}_{4}: \mathrm{Tm}^{3+} 0.5 \mathrm{~mol} \%, \mathrm{Yb}^{3+} 30 \mathrm{~mol} \% @ \mathrm{NaYF}_{4}$ core/shell UCNPs; (b) emission spectra of UCNPs under $980 \mathrm{~nm}$ NIR excitation (8 W).

the more $o$-NB groups there are the lower LCST the block copolymer exhibits [33]. Therefore, the block copolymers in current work would be amphiphilic and able to self-assemble to micelles at room temperature. In this work, we used pyrene as the probe to investigate self-assembly behaviors of the block copolymers at room temperature. Figure 2 shows plots of the ratio of fluorescent intensities of pyrene at $336 \mathrm{~nm}$ and $332.5 \mathrm{~nm}\left(I_{336} / I_{332.5}\right)$ versus concentrations of the block copolymers. The abrupt rises of intensity ratios indicate that the block copolymers self-assemble in water at room temperature, and the corresponding concentrations show that the critical micelle concentrations (CMCs) of $\mathrm{pl}$ and p2 are about $0.24 \mathrm{~g} / \mathrm{L}$ and $0.056 \mathrm{~g} / \mathrm{L}$, respectively. The CMC of $\mathrm{p} 1$ is about 4 times the one of $\mathrm{p} 2$, indicating that the $\mathrm{P}$ (NIPAM-co-NBA) block of $\mathrm{p} 1$ is more hydrophobic because of its more NBA moieties comparing to the one of $\mathrm{p} 2$. The self-assemblies were further observed by TEM and DLS. As shown in Figures 3(a) and 3(b), the self-assemblies of p1 and 
p2 are spherical micelles with the diameters about $115.7 \mathrm{~nm}$ and $140.5 \mathrm{~nm}$, respectively, which coincide with the results from DLS (insets in Figures 3(a) and 3(b)).

$o$-NB esters are a typical photolabile group, which usually yields carboxylic acid under irradiation of UV light with $o$ nitrosobenzaldehyde as a byproduct [11]. When P(NIPAMco-NBA) are treated with UV light, they will convert to $\mathrm{P}$ (NIPAM-co-AA) (Scheme 1), where the AA denotes acrylic acid. The LCST of P(NIPAM-co-AA) is higher than $40^{\circ} \mathrm{C}$ even when AA content is just $5 \mathrm{~mol} \%$ [33]. That is, the UV light will convert the amphiphilic block copolymers to hydrophilic ones at room temperature and cause the disintegration of the corresponding micelles. To verify the UV light induced photocleavage reactions of the $o$-NB esters in this work, the UV-vis absorption measurements were performed. Figures 4(a) and 4(b) show the UV-vis absorption spectra of the micellar solutions of $\mathrm{p} 1$ and $\mathrm{p} 2$ irradiated by UV light $\left(365 \mathrm{~nm}, 2000 \mathrm{~mW} / \mathrm{cm}^{2}\right)$ for various time. The decrease in absorption at $265 \mathrm{~nm}$ and the increase at $313 \mathrm{~nm}$ prove cleavage of photolabile $o$-nitrobenzyl ester moieties and the generation of $o$-nitrosobenzaldehyde [21, 34]. Disintegration of the micelles was able to be observed directly. For example, the original aqueous solution of $\mathrm{pl}$ was turbid and exhibited strong Tyndall effect (Figure 4(c)); however, when the p1 solution was treated by UV light for $7 \mathrm{~min}$, it became transparent and its Tyndall effect nearly disappeared, indicating the disintegration of the micelles.

4.3. Photocontrolled Release upon UV Irradiation. The micelles with the character of UV-triggered disintegration may be used as drug carriers with the character of photocontrolled drug release. In this work, we investigated the UV-triggered drug release from the micelles using Nile Red (NR) as the model compound. The principle of using $\mathrm{NR}$ as a model compound is based on the fact that the fluorescence emission of NR is low in water due to its very low solubility but becomes much more intense when NR is solubilized in hydrophobic micelle core $[37,38]$. Figure 5(a) shows the spectral change of the micellar solution of $\mathrm{p} 1$ exposed to UV light for various time. It can be seen that the fluorescent intensity of NR gradually decreases with the increase of the UV irradiation time, indicating that the NR were released from the hydrophobic micelle core to the aqueous solution. Furthermore, we investigated the influence of $o$-NB ester content on the kinetics of NR release. Figure 5(b) shows the plots of normalized fluorescent intensities versus irradiation time for the NR loaded micelles of the two block copolymers. It can be seen that the less the NBA moieties the polymer has, the faster the fluorescence decreases. Therefore, it can be deduced that the micelles self-assembled by the block copolymer with less NBA content requiring less irradiation time of light to release the cargoes.

4.4. Preparation of the NIR Responsive Hybrid Nanoparticles and the NIR-Induced Release. To convert NIR to UV light, $\mathrm{NaYF}_{4} \mathrm{Tm}^{3+} \mathrm{Yb}^{3+}$ UCNPs were prepared. The morphologies observed by TEM revealed that the particles were nearly ellipsoid with major axis and minor axis about $36 \mathrm{~nm}$ and

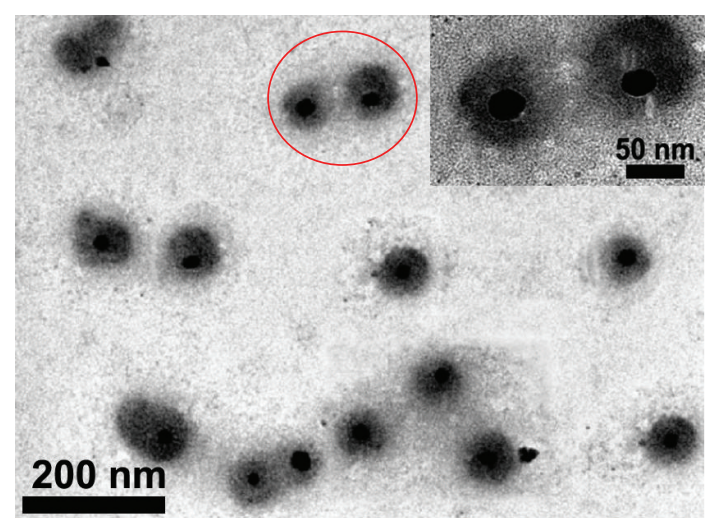

FIGURE 7: High-resolution TEM micrograph of the hybrid nanoparticles. Inset is the magnified image of the region in red circle. Note. Staining was performed with phosphotungstic acid.

$24 \mathrm{~nm}$, respectively (Figure 6(a)). The fluorescent spectrum of the UCNPs excited by NIR light at $980 \mathrm{~nm}$ showed output of UV light at about $365 \mathrm{~nm}$ (Figure 6(b)), indicating that the UCNPs might be used to trigger the photocleavage reaction of the $o$-NB groups. In this work, we prepared hybrid nanoparticles using $\mathrm{p} 1$ as the organic part. TEM observation indicated that the hybrid nanoparticles were about $120 \mathrm{~nm}$ and exhibited a core-shell structure with UCNP as the core and polymers as the corona (Figure 7). To investigate the potential of the hybrid nanoparticles as carriers with the character of NIR-induced drug release, we loaded NR in the hybrid nanoparticles and investigated the NR release under irradiation of NIR light. As shown in Figure 8(a), the fluorescence of the NR loaded hybrid nanoparticles gradually decreases with the varying irradiation time of NIR. However, when NR were loaded in micelles of pl, there was nearly no change in the fluorescence intensity of NR after irradiation of NIR light (Figure $8(\mathrm{~b})$ ). The results proved that the hybrid nanoparticles released NR by the stimulus of NIR.

\section{Conclusion}

In conclusion, we synthesized UCNPs and thermo- and UV light-responsive block copolymers. The UCNPs were able to convert NIR light at $980 \mathrm{~nm}$ to UV light at $365 \mathrm{~nm}$, and the sensitivity of the polymers to UV light was able to be controlled by the content of the photocleavable moieties. By self-assembling the polymers with the presence of the UCNPs in water, core-shell hybrid nanoparticles with the UCNP as the core and the polymers as the shell were obtained. Using NR as the model compound, the hybrid nanoparticles were proved to be potential drug carriers with the character of the NIR-triggered drug release.

\section{Conflict of Interests}

The authors declare that there is no conflict of interests regarding the publication of this paper. 


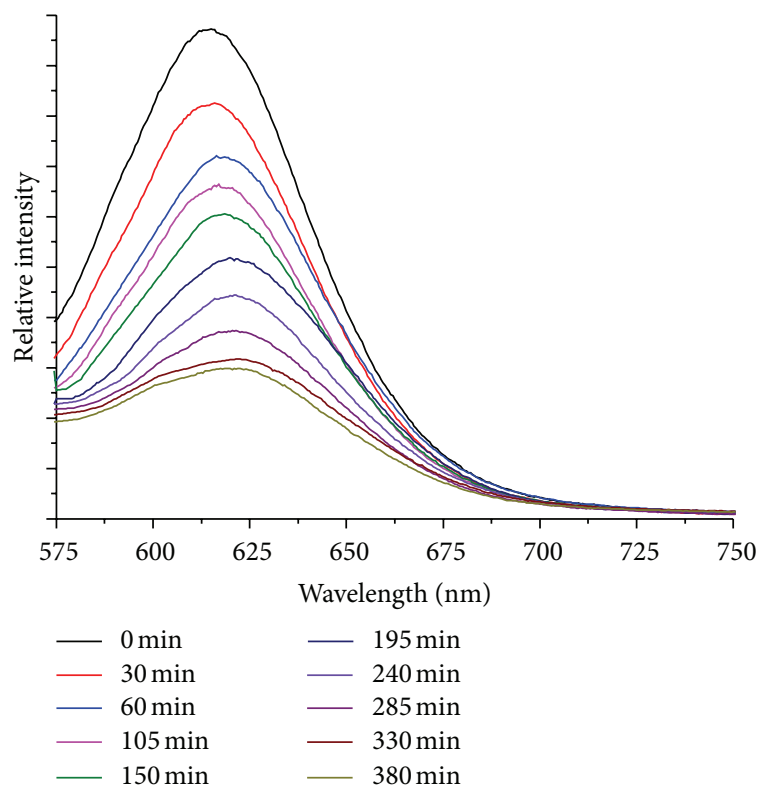

(a)

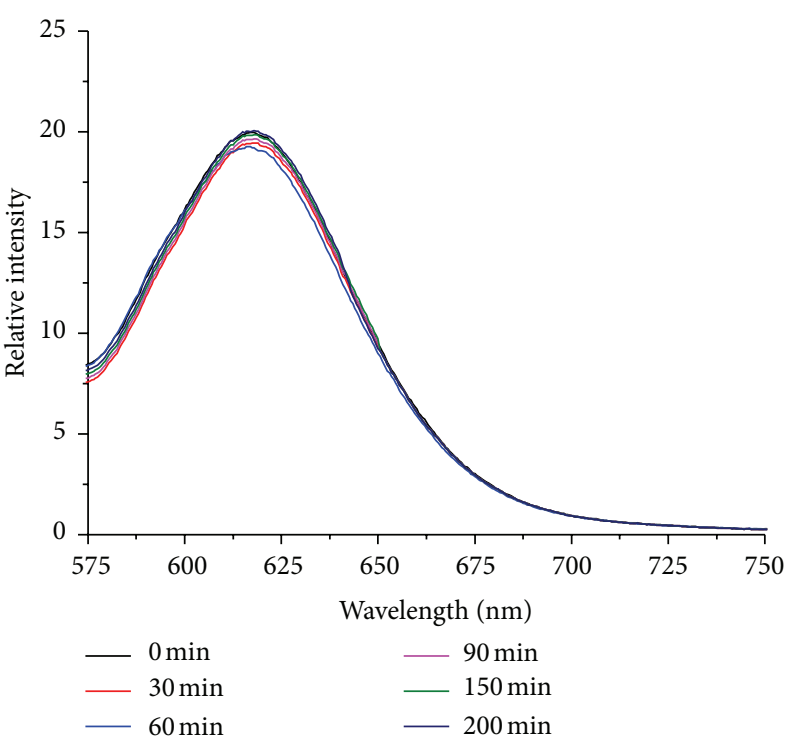

(b)

FIGURE 8: Fluorescence emission spectra $\left(\lambda_{\text {ex }}=550 \mathrm{~nm}\right.$ ) of the p1 micellar solution (a) loaded with both UCNPs and Nile Red; (b) with only encapsulated Nile Red under $980 \mathrm{~nm}$ NIR light $(8 \mathrm{~W})$.

\section{Acknowledgments}

This work is supported by the National Natural Scientific Foundation of China Project (21304028 and 51203145), the Fundamental Research Funds for the Central Universities (2013HGCH0001), and the Student Innovation Training Program of Hefei University of Technology (2013CXSY722).

\section{References}

[1] R. Lehner, X. Y. Wang, M. Wolf, and P. Hunziker, "Designing switchable nanosystems for medical application," Journal of Controlled Release, vol. 161, no. 2, pp. 307-316, 2012.

[2] P. Schattling, F. D. Jochum, and P. Theato, "Multi-stimuli responsive polymers-the all-in-one talents," Polymer Chemistry, vol. 5, no. 1, pp. 25-36, 2014.

[3] S. Mura, J. Nicolas, and P. Couvreur, "Stimuli-responsive nanocarriers for drug delivery," Nature Materials, vol. 12, no. 11, pp. 991-1003, 2013.

[4] A. Chan, R. P. Orme, R. A. Fricker, and P. Roach, "Remote and local control of stimuli responsive materials for therapeutic applications," Advanced Drug Delivery Reviews, vol. 65, no. 4, pp. 497-514, 2013.

[5] R. Cheng, F. H. Meng, C. Deng, H. A. Klok, and Z. Y. Zhong, "Dual and multi-stimuli responsive polymeric nanoparticles for programmed site-specific drug delivery," Biomaterials, vol. 34, no. 14, pp. 3647-3657, 2013.

[6] J. Gohy and Y. Zhao, "Photo-responsive block copolymer micelles: design and behavior," Chemical Society Reviews, vol. 42, no. 17, pp. 7117-7129, 2013.

[7] N. Fomina, J. Sankaranarayanan, and A. Almutairi, "Photochemical mechanisms of light-triggered release from nanocarriers," Advanced Drug Delivery Reviews, vol. 64, no. 11, pp. 10051020, 2012.
[8] W. Cao, Y.-W. Gu, M. Meineck, and H.-P. Xu, “The combination of chemotherapy and radiotherapy towards more efficient drug delivery," Chemistry, vol. 9, no. 1, pp. 48-57, 2014.

[9] B. Wang, K.-F. Chen, R.-D. Yang, and F. Yang, "Stimulusresponsive polymeric micelles for the light-triggered release of drugs," Carbohydrate Polymers, vol. 103, pp. 510-519, 2014.

[10] Q.-J. Xing, N.-J. Li, and D.-Y. Chen, "Light-responsive amphiphilic copolymer coated nanoparticles as nanocarriers and realtime monitors for controlled drug release," Journal of Materials Chemistry B, vol. 2, no. 9, pp. 1182-1189, 2014.

[11] H. Zhao, E. S. Sterner, E. B. Coughlin, and P. Theato, "ONitrobenzyl alcohol derivatives: opportunities in polymer and materials science," Macromolecules, vol. 45, no. 4, pp. 1723-1736, 2012.

[12] L.-L. Meng, W. Huang, D.-L. Wang, X.-H. Huang, X.-Y. Zhu, and D.-Y. Yan, "Chitosan-based nanocarriers with $\mathrm{pH}$ and light dual response for anticancer drug delivery," Biomacromolecules, vol. 14, no. 8, pp. 2601-2610, 2013.

[13] Y. Liu, C. Y. Yu, H. B. Jin et al., "A supramolecular Janus hyperbranched polymer and its photoresponsive self-assembly of vesicles with narrow size distribution," Journal of the American Chemical Society, vol. 135, no. 12, pp. 4765-4770, 2013.

[14] Y. Zhao, "Light-responsive block copolymer micelles," Macromolecules, vol. 45, no. 9, pp. 3647-3657, 2012.

[15] T. Furuta, S. S.-. Wang, J. L. Dantzker et al., "Brominated 7hydroxycoumarin-4-ylmethyls: photolabile protecting groups with biologically useful cross-sections for two photon photolysis," Proceedings of the National Academy of Sciences of the United States of America, vol. 96, no. 4, pp. 1193-1200, 1999.

[16] Z.-J. Zhang, J. Wang, and C.-Y. Chen, "Near-infrared lightmediated nanoplatforms for cancer thermo-chemotherapy and optical imaging," Advanced Materials, vol. 25, no. 28, pp. 38693880, 2013. 
[17] J. You, P.-Z. Zhang, F.-Q. Hu et al., "Near-infrared light-sensitive liposomes for the enhanced photothermal tumor treatment by the combination with chemotherapy," Pharmaceutical Research, vol. 31, no. 3, pp. 554-565, 2014.

[18] H.-S. Qian and Y. Zhang, "Synthesis of hexagonal-phase coreshell $\mathrm{NaYF}_{4}$ nanocrystals with tunable upconversion fluorescence," Langmuir, vol. 24, no. 21, pp. 12123-12125, 2008.

[19] H. S. Qian, H. C. Guo, P. C. Ho, R. Mahendran, and Y. Zhang, "Mesoporous-silica-coated up-conversion fluorescent nanoparticles for photodynamic therapy," Small, vol. 5, no. 20, pp. 2285-2290, 2009.

[20] X. Liu, H. Qian, Y. Ji et al., "Mesoporous silica-coated $\mathrm{NaYF}_{4}$ nanocrystals: facile synthesis, in vitro bioimaging and photodynamic therapy of cancer cells," RSC Advances, vol. 2, no. 32, pp. 12263-12268, 2012.

[21] B. Yan, J. C. Boyer, N. R. Branda, and Y. Zhao, "Near-infrared light-triggered dissociation of block copolymer micelles using upconverting nanoparticles," Journal of the American Chemical Society, vol. 133, no. 49, pp. 19714-19717, 2011.

[22] T.-Q. Wu, M. Barker, K. M. Arafeh, J. C. Boyer, C. J. Carling, and P. N. Branda, "A UV-blocking polymer shell prevents one-photon photoreactions while allowing multi-photon processes in encapsulated upconverting nanoparticles," Angewandte Chemie-International Edition, vol. 125, no. 42, pp. 11312-11315, 2013.

[23] Y.-Z. Min, J.-M. Li, F. Liu, E. L. Yeow, and B.-G. Xing, "Nearinfrared light-mediated photoactivation of a platinum antitumor prodrug and simultaneous cellular apoptosis imaging by upconversion-luminescent nanoparticles," Angewandte Chemie, vol. 126, no. 4, pp. 1030-1034, 2014.

[24] T.-Q. Wu, M. Barker, K. M. Arafeh, J. C. Boyer, C. J. Carling, and P. N. Branda, "A UV-blocking polymer shell prevents one-photon photoreactions while allowing multi-photon processes in encapsulated upconverting nanoparticles," Angewandte Chemie International Edition, vol. 125, no. 42, pp. 1131211315, 2013.

[25] Y.-M. Yang, B. Velmurugan, X.-G. Liu, and B.-G. Xing, "NIR photoresponsive crosslinked upconverting nanocarriers toward selective intracellular drug release," Small, vol. 9, no. 17, pp. 2937-2944, 2013.

[26] M. Bikram and J. L. West, "Thermo-responsive systems for controlled drug delivery," Expert Opinion on Drug Delivery, vol. 5, no. 10, pp. 1077-1091, 2008.

[27] S. H. Choi, S. H. Lee, and T. G. Park, “Temperature-sensitive pluronic/poly(ethylenimine) nanocapsules for thermally triggered disruption of intracellular endosomal compartment," Biomacromolecules, vol. 7, no. 6, pp. 1864-1870, 2006.

[28] E. S. Lee, Z. Gao, and Y. H. Bae, "Recent progress in tumor $\mathrm{pH}$ targeting nanotechnology," Journal of Controlled Release, vol. 132, no. 3, pp. 164-170, 2008.

[29] D.-Q. Chen and H.-B. Wang, "Novel pH-sensitive biodegradable polymeric drug delivery systems based on ketal polymers," Journal of Nanoscience and Nanotechnology, vol. 14, no. 1, pp. 983-989, 2014.

[30] T. Xing, B. Lai, and L.-F. Yan, "Disulfide cross-linked polypeptide nanogel conjugated with a fluorescent probe as a potential image-guided drug-delivery agent," Macromolecular Chemistry and Physics, vol. 214, no. 5, pp. 578-588, 2013.

[31] J. Liu, Y. Pang, W. Huang et al., "Redox-responsive polyphosphate nanosized assemblies: a smart drug delivery platform for cancer therapy," Biomacromolecules, vol. 12, no. 6, pp. 24072415, 2011.
[32] H. Wei, S. X. Cheng, X. Z. Zhang, and R. X. Zhuo, "Thermosensitive polymeric micelles based on poly( $\mathrm{N}$-isopropylacrylamide) as drug carriers," Progress in Polymer Science, vol. 34, no. 9, pp. 893-910, 2009.

[33] L. Ionov and S. Diez, "Environment-friendly photolithography using poly( $N$-isopropylacrylamide)-based thermoresponsive photoresists," Journal of the American Chemical Society, vol. 131, no. 37, pp. 13315-13319, 2009.

[34] Y.-M. Li, Y.-F. Qian, T. Liu, G.-Y. Zhang, and S.-Y. Liu, "Lighttriggered concomitant enhancement of magnetic resonance imaging contrast performance and drug release rate of functionalized amphiphilic diblock copolymer micelles," Biomacromolecules, vol. 13, no. 11, pp. 3877-3886, 2012.

[35] L.-F. Yan and W. Tao, "One-step synthesis of pegylated cationic nanogels of poly $\left(N, N^{\prime}\right.$-dimethylaminoethyl methacrylate) in aqueous solution via self-stabilizing micelles using an amphiphilic macroRAFT agent," Polymer, vol. 51, no. 10, pp. 2161-2167, 2010.

[36] J. Du, L. Tang, W. Song, Y. Shi, and J. Wang, "Evaluation of polymeric micelles from brush polymer with poly( $\varepsilon$-caprolactone)b-poly(ethylene glycol) side chains as drug carrier," Biomacromolecules, vol. 10, no. 8, pp. 2169-2174, 2009.

[37] A. P. Goodwin, J. L. Mynar, Y. Ma, G. R. Fleming, and J. M. J. Fréchet, "Synthetic micelle sensitive to IR light via a two-photon process," Journal of the American Chemical Society, vol. 127, no. 28, pp. 9952-9953, 2005.

[38] J. Q. Jiang, X. Tong, D. Morris, and Y. Zhao, “Toward photocontrolled release using light-dissociable block copolymer micelles," Macromolecules, vol. 39, no. 13, pp. 4633-4640, 2006. 

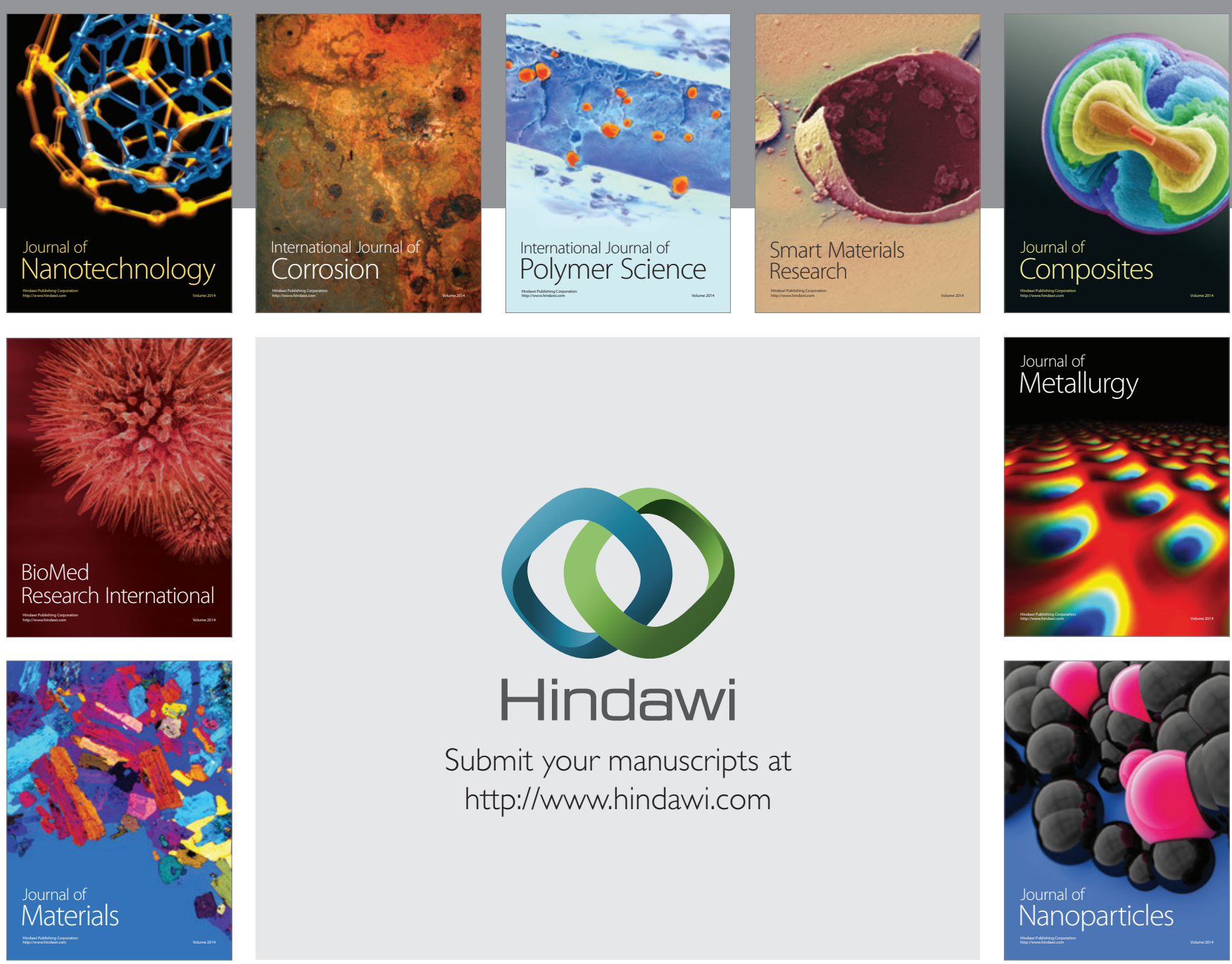

Submit your manuscripts at http://www.hindawi.com
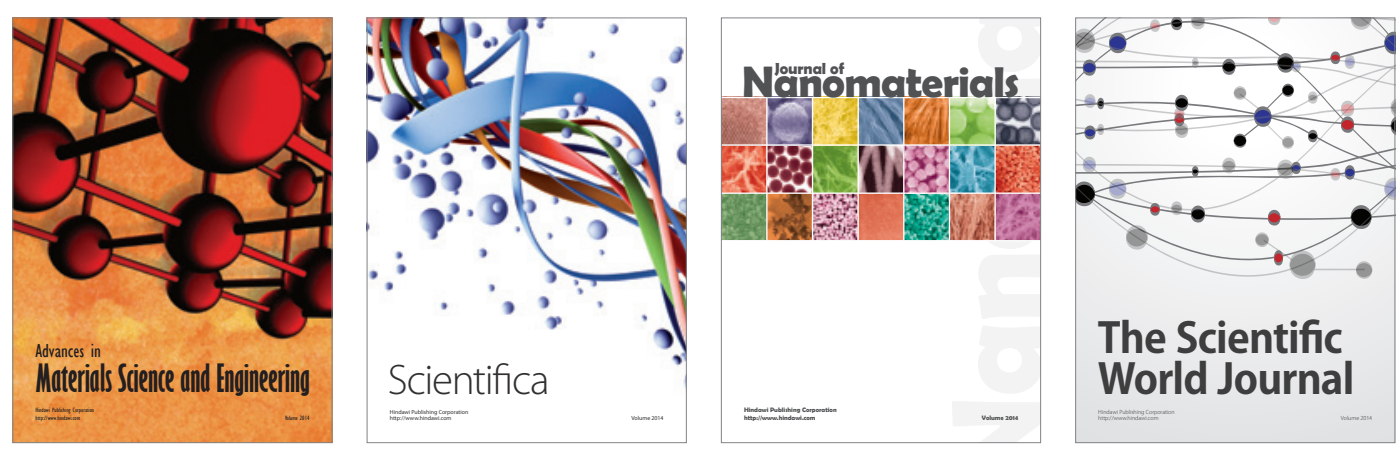

\section{The Scientific World Journal}
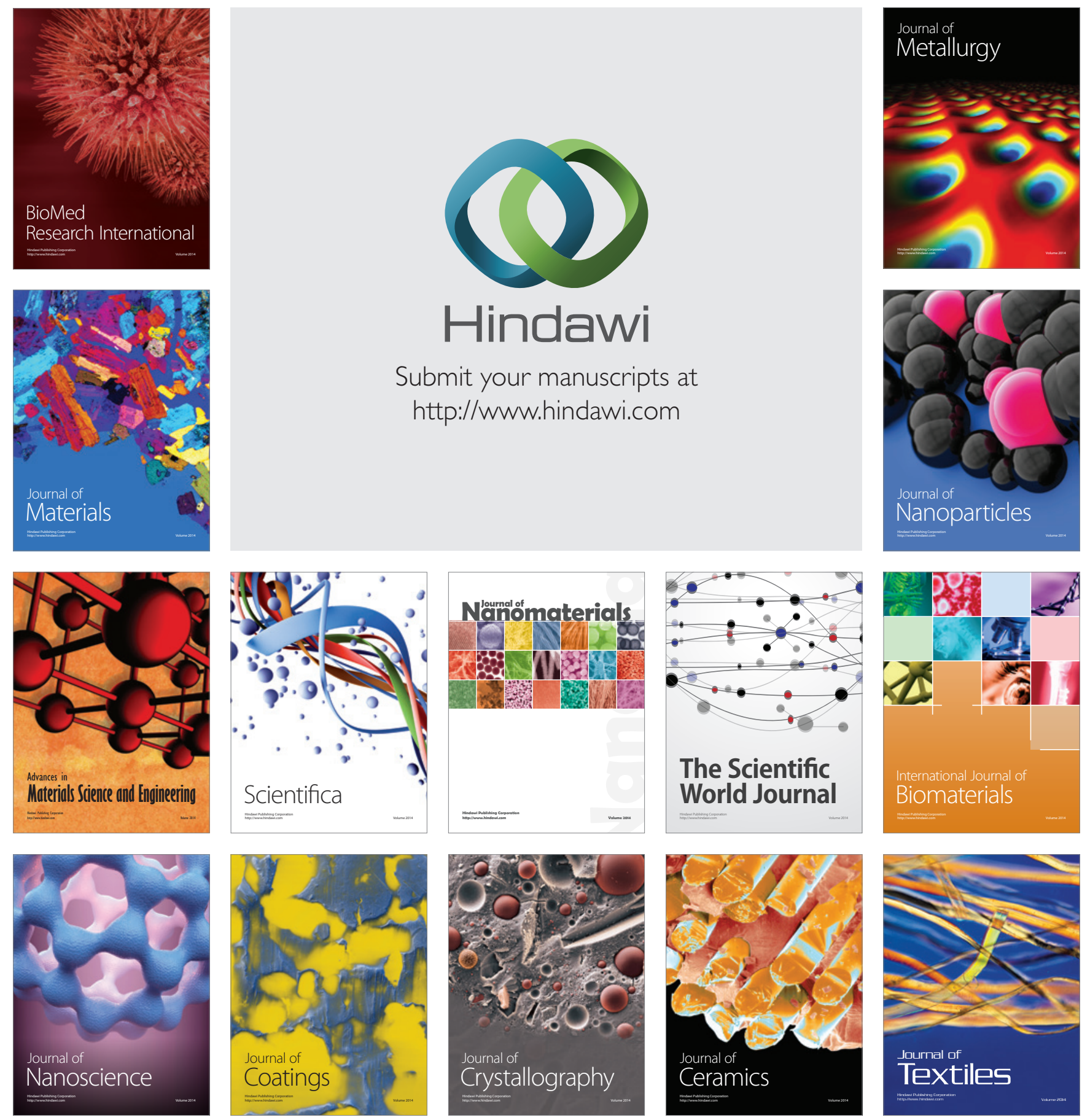